\title{
IAPURDUM
}

Euskal ikerketen aldizkaria | Revue d'études basques |

Revista de estudios vascos | Basque studies review

$1 \mid 1996$

Numéro I

\section{Compte-rendu du mémoire de maîtrise en histoire d'Anne Delpeuch}

Josette Pontet

\section{(2) OpenEdition}

Journals

Édition électronique

URL : http://journals.openedition.org/lapurdum/1912

DOI : 10.4000/lapurdum.1912

ISSN : 1965-0655

Éditeur

IKER

Édition imprimée

Date de publication : 1 octobre 1996

Pagination : 229-233

ISBN : 2-84127-106-4

ISSN : $1273-3830$

Référence électronique

Josette Pontet, «Compte-rendu du mémoire de maîtrise en histoire d'Anne Delpeuch », Lapurdum [En ligne], 1 | 1996, mis en ligne le 01 septembre 2010, consulté le 30 janvier 2020. URL : http:// journals.openedition.org/lapurdum/1912 ; DOI : 10.4000/lapurdum.1912 


\section{COMPTE-RENDU DU MÉMOIRE DE MAÎTRISE EN HISTOIRE D'ANNE DELPEUCH}

Compte-rendu du travail d'étude et de recherche préparé par Anne Delpeuch, sous la direction du professeur Josette Pontet, Université Michel-de-Montaigne Bordeaux III, sujet : Étude démographique d'unè société coutumière. Ustaritz de 1745 à 1850, 2 tomes dactylographiés, t1, 185 pages de texte et annexes, t2, tableaux de résultats fournis par logiciel CASOAR, 200 p.

Le mémoire soutenu par Anne Delpeuch pour l'obtention de sa maîtrise d'histoire est un travail important par l'ampleur de sa documentation, par la nouveauté de la méthode employée (reconstitution des familles), par les résultats obtenus qui éclairent d'une manière neuve les caractéristiques démographiques de la population labourdine d'Ustaritz. Le principe de l'étude repose sur le dépouillement systématique des registres paroissiaux et d'état civil de la commune d'Ustaritz, dans l'ensemble assez bien conservés : les premiers registres remontent à 1600 pour Ustaritz et à 1671 pour Arrauntz, mais les séries continues ne commencent vraiment qu'après 1740 et c'est à partir de cette date qu'a été entreprise la reconstitution informatisée des familles grâce au logiciel CASOAR mis au point par J.-P. Bardet et M. Hainsworth, et amélioré par J. Renard, dans le but principal de mesurer la fécondité des familles du passé. Dix mille actes environ ont été traités qui ont permis la reconstitution de plus de 2000 familles entre le milieu du XVIII ${ }^{\text {e }}$ siècle et le milieu du XIX ${ }^{e}$ siècle, dont cependant ne peuvent être utilisées que $59 \%$ pour une étude fine de la fécondité des femmes. On ne se propose ici que de donner les principaux éléments de ce travail. Ils montrent de réelles originalités de la société uztariztar, mais par bien des côtés aussi, cette société a des comportements démographiques que l'on retrouve aux mêmes dates, dans le Sud-Ouest de la France.

Comme partout, on ne se marie pas pendant les temps clos de l'Avert (de la Saint-André 30 novembre à l'Épiphanie) et du Carême. Très peu de mariages sont enregistrés en décembre et en mars ; mais on se marie très peu également en mai sans que l'on puisse bien expliquer la désaffection des époux, que l'on retrouve ailleurs pour ce mois. En fait les mariages sont très concentrés sur les mois de février, novembre et janvier qui regroupent $70 \%$ des mariages célébrés, alors que l'on se marie peu pendant les mois d'été consacrés aux travaux des champs. Ce 
calendrier est respecté à peu près pendant toute la période, à quelques nuances près, dont une importante concernant la Révolution : entre 1790 et 1814, la déchristianisation est à l'œuvre, et en tout cas les temps clos ne sont plus scrupuleusement respectés : en indice on passe pendant l'Avent de 0 à 45 et pendant le Carême de 12 à 53 . Avec la Restauration et la Monarchie de Juillet, la reprise en main de l'Église est évidente et, en 1840-1849, les indices de l'Avent et du Carême sont respectivement à 9 et 13 , fort proches de ce qu'ils étaient sous l'Ancien Régime. La perturbation révolutionnaire touche aussi les jours choisis pour se marier. En effet jusqu'en 1789, le jour de très loin le plus choisi est le mardi qui voit se célébrer plus des deux tiers des mariages, habitude que l'on retrouve dans l'ensemble de la Gascogne ; viennent ensuite le mercredi $(12 \%)$ et le samedi $(11 \%)$. Dimanche et vendredi sont presque totalement délaissés. Or en 1790-1814, on a une totale redistribution des jours : le mardi n'est plus choisi que par moins d'un tiers des époux, alors que le dimanche l'est par $17 \%$ et le vendredi par $8 \%$, les autres jours de la semaine se partageant presque également les mariages, sauf le lundi qui est désormais à égalité avec le dimanche. Mais le retour à la monarchie ne se traduit pas ici par un simple retour au passé : certes la place du dimanche régresse, mais le mardi ne retrouve pas sa prédominance : il est même dépassé par le mercredi en 1840-1849, et c'est désormais sur trois jours : mercredi, mardi et lundi que se célèbrent les deux tiers des mariages, jeudi et vendredi restant des jours presque complètement délaissés.

On peut noter encore une légère évolution au cours du XIX ${ }^{c}$ siècle : une progression limitée des mois d'été mais qui laisse ces derniers encore très loin dans les choix des conjoints. On constate d'ailleurs que les artisans se marient davantage l'été que les agriculteurs et qu'ils respectent moins les temps clos que ceux-ci.

Ce qui distingue particulièrement les uztariztars c'est l'âge très tardif au mariage, aussi bien pour les hommes que pour les femmes, que l'on retrouve sur l'ensemble de la période avec des variations au demeurant assez faibles: l'homme se marie en moyenne à un peu plus de 32 ans, la femme à presque 30 ans. L'âge au mariage a augmenté aussi bien pour les hommes que pour les femmes de 1765 à 1815, après, l'âge des femmes a tendance à baisser légèrement mais reste encore très élevé si on le compare à ce que l'on connaît ailleurs : la différence est de plus de 3 ans avec les moyennes nationales, mais il est également plus élevé qu'à Orthez où il dépasse 25 ans dans la première moitié du $\mathrm{XIX}^{\bullet}$ siècle. Il est bien évident que cela n'est pas sans incidence sur la fécondité. Ces âges tardifs sont sans doute à mettre en relation avec le droit successoral et la cohabitation imposée entre maîtres anciens et maîtres jeunes, cohabitation que les parents retardent visiblement le plus possible. Il aurait été intéressant de distinguer entre les âges des héritier(e)s et des cadet(te)s, malheureusement cela n'a pas été pris en compte dans le mémoire.

Comme partout la norme est que l'homme soit plus âgé que la femme, et cette norme tend à s'affirmer après la Révolution : l'homme est plus âgé dans $55 \%$ des mariages entre 1765-1789, et dans près des 
deux tiers en 1815-1849; comme partout, on n'épouse guère une femme de son âge (environ $7 \%$ ) ; mais dans l'ensemble les écarts d'âge entre homme et femme sont assez peu importants, même si là encore ils augmentent du $\mathrm{XVIII}^{c}$ au $\mathrm{XIX}^{e}$ siècle passant en moyenne de 2,31 à presque 3 ans.

La pratique du remariage est assez peu répandue à Uztaritz et ne représente qu'un peu plus de $6 \%$ du total des mariages; les veuves ne se remarient que rarement, il n'y a que $2,5 \%$ des mariages à compter une veuve, mais le pourcentage n'atteint que $5 \%$ pour les veufs qui n'épousent qu'exceptionnellement une veuve.

Ce n'est pas une réelle surprise, mais la société d'Ustaritz apparaît fort peu alphabétisée, si l'on retient, comme on le fait habituellement le critère des signatures au mariage, et le décalage entre hommes et femmes est très marqué. En effet, environ $30 \%$ des époux signent leur acte de mariage au XVIII' siècle; les progrès sont réels après 1820 et, en 1840-1849, la moitié des époux sont capables d'apposer leur signature en se mariant. Mais à la même époque ce sont plus de $80 \%$ des mariés bayonnais qui signent leur acte de mariage. L'analphabétisme touche au XVIII' siècle près de $95 \%$ des épouses à Ustaritz et les progrès sont très lents à se dessiner : en 1840-1849 il n'y a encore qu'une épouse sur cinq à mettre son nom au bas de l'acte de mariage. Cette mise à l'écart des filles de l'instruction primaire peut paraître surprenante dans un pays qui leur reconnaît le droit d'hériter et donc de gérer le patrimoine ; mais l'inégalité devant le savoir est patente.

Derrière ces pourcentages se cachent comme ailleurs d'autres inégalités : celles qui tiennent au milieu socioprofessionnel : les notables ainsi que leurs épouses sont très largement alphabétisés (tous les époux et les trois quarts des épouses signent) les artisans sont assez bien alphabétisés (près de six sur dix signent), mais leurs femmes ne le sont pas (une sur dix seulement sait signer); quant aux agriculteurs, ils sont fort peu alphabétisés (18\%) et leurs femmes encore moins $(7 \%)$.

Or Ustaritz compte une part très importante de sa population travaillant la terre : à partir des professions déclarées au mariage, $43 \%$ des hommes et $46 \%$ des femmes exercent un métier agricole (dont $14 \%$ de métayères). L'appellation la plus répandue est celle de laboureur chez les hommes et de cultivatrice chez les femmes. Le secteur artisanal regroupe $27,5 \%$ des professions déclarées, avec cinq secteurs s'équilibrant à peu près, bâtiment (beaucoup de tuiliers), bois (beaucoup de charpentiers), cuir (nombreux cordonniers et corroyeurs), alimentation (avec nombre de boulangers et de chocolatiers), textile (avec une majorité de tisserands). Chez les femmes seul ce secteur et représenté, (avec un nombre assez important de couturières. Une des particularités d'Ustaritz est de compter un nombre important de douaniers, soldats, gendarmes : ils représentent plus de $10 \%$ des époux. Ce qui frappe aussi c'est la faiblesse du secteur marchand dans un secteur tertiaire où les hommes de loi (au XVIII siècle surtout), et les professions libérales sont un peu mieux représentées ( $6 \%$ des époux), ainsi que les métiers 
des transports (4\%). Cette configuration socioprofessionnelle donne à l'évidence à Ustaritz plus les caractères d'un bourg rural que d'une petite ville, en dépit de ses fonctions judiciaires et du siège du Biltzar. Celles-ci expliquent cependant la présence d'une domesticité féminine un peu nombreuse ( $5 \%$ des épouses) mais qui n'a rien de comparable à ce que l'on peut trouver à Bayonne à la même époque. Au bas de l'échelle sociale apparaissent mendiants et mendiantes (5\% des époux) et bohémiens et bohémiennes (1\%). Comme partout, c'est seulement à partir du XIX ${ }^{e}$ siècle que sont mentionnées les activités féminines, mais une femme sur cinq se qualifie seulement de ménagère.

Il est intéressant de mesurer l'attractivité d'Ustaritz sur sa région : près de la moitié des hommes qui s'y marient n'y sont pas nés $(44,5 \%)$, mais beaucoup sont venus avant le mariage : en effet, $85 \%$ des époux y résident ; quant aux femmes, un tiers d'entre elles ne sont pas natives d'Ustaritz, mais près de $90 \%$ y résident au moment de leur mariage.

Une grande part des époux et des épouses non natifs d'Ustaritz vient du Labourd : celui-ci fournit la moitié des hommes les deux tiers des femmes. Vient ensuite la Basse-Navarre $(22,5 \%$ des hommes et $25 \%$ des femmes); en revanche la Soule est très faiblement représentée (moins que le Béarn pour les hommes). L'aire d'attraction d'Ustaritz apparaît ainsi assez nettement circonscrite aux villages les plus proches; elle n'exclut pas pourtant la venue d'époux plus lointains d'Espagne notamment, et particulièrement du Guipuzcoa, mais aussi d'un certain nombre de départements français du Sud-Ouest ou plus éloignés, la présence de douaniers et de militaires expliquant en partie ce recrutement hexagonal.

Une des questions essentielles posées par ce mémoire est celle de la fécondité de la population que nous venons de décrire. Il s'intéresse d'abord au calendrier des conceptions : moins irrégulier que celui des mariages, il fait néanmoins apparaître des variations mensuelles assez marquées : peu de conceptions en mars, en liaison avec le jeûne du Carême, peu de conceptions d'août à décembre, mais des mois plus féconds : janvier, février, avril, mai, juin. Une approche grossière des descendances en calculant le quotient naissances-mariages montre un niveau assez bas sur l'ensemble de la période : 4,14 avec une baisse du quotient dans la deuxième moitié du XVIII ${ }^{e}$ siècle (3,34 en 1795-1809) avant une remontée sous la Restauration et une légère baisse ensuite.

Létude fine de la fécondité à la fois par analyse longitudinale et transversale confirme en gros cette évolution. Il n'est pas question d'entrer ici dans les détails des calculs et des tableaux fort précis concernant la fécondité des femmes selon leur âge au mariage, la durée du mariage. Nous nous contenterons de noter que comme partout le taux de fécondité diminue avec l'âge grandissant de la femme, mais ici se pose avec acuité le problème de l'âge tardif au mariage qui explique le nombre finalement peu élevé d'enfants par femme, mais aussi le maintien d'une fécondité plus soutenue encore après 35 ans, ce que l'on ne trouve pas dans d'autres régions. Une des questions posées est de savoir s'il y a évo- 
lution vers une fécondité volontairement contrôlée. La réponse n'est ni simple ni nette : celle-ci paraît effective pour les femmes ayant 25-29 ans, qui ont un taux de fécondité en baisse entre 1765-1789 (657) et 1815-1824 (388). Mais cela n'est plus perceptible pour les femmes plus âgées et l'âge de la dernière maternité tend à augmenter, sans doute en liaison avec l'âge de plus en plus tardif au mariage mais aussi parce qu'il y a une reprise de la fécondité à partir de la Restauration : on pourrait alors poser la question d'un meilleur encadrement de l'Église ; toutefois d'autres explications peuvent être trouvées à ce comportement qui n'est pas exceptionnel : on a montré ailleurs une reprise de la fécondité à l'époque de la Restauration et de la Monarchie de Juillet. Cela pourrait cependant aller dans le sens d'un plus grand contrôle social : le pourcentage de conceptions prénuptiales qui était de $19 \%$ en $1765-1784$, de $26 \%$ en $1785-1804$, de $25 \%$ en $1805-1824$, n'est que de $20 \%$ en 1825-1844.

Quoi qu'il en soit, les familles d'Ustaritz sont dans l'ensemble assez peu nombreuses : $9 \%$ n'ont pas d'enfants, le tiers a de 1 à 3 enfants, un autre gros tiers a entre 4 et 6 enfants, mais les familles de 10 enfants et plus représentent moins de $2 \%$ des cas, alors que plus de la moitié des familles qui ont des enfants en ont 4 au plus.

S'il y a des mois pour se marier ou naître, il y a aussi des mois pour mourir à Ustaritz comme ailleurs. On distingue deux pointes de mortalité dans l'année : elles concernent l'hiver (janvier/février) et l'automne avec des poussées dès la fin de l'été : avril, septembre, octobre concentrent $30 \%$ des décès au XVIII siècle. Comme partout la mortalité d'été/automne régresse au XIX ${ }^{c}$ siècle, restant cependant forte pour les enfants, lorsque la mortalité d'hiver (janvier/février/mars) augmente. La mortalité infantile (quotients corrigés) est de $240 \%$ au XVIII' siècle, mais se maintient à un niveau très élevé au XIX ${ }^{\circ}$ siècle : elle serait de $367 \%$ on 1823-1832. Sur l'ensemble de la période, sur 1000 enfants nés, 666 sont encore vivants à 14 ans, ce qui représente un assez bon taux de survie. Quant à la mortalité des femmes en couche, elle paraît toucher $6 \%$ des mères.

Voilà donc éclairé un certain nombre de caractéristiques de la population d'Ustaritz par ce travail tout à fait considérable dans le cadre d'une mâitrise réalisé par Anne Delpeuch. Certes, bien des éléments restent à approfondir ou des analyses à affiner, mais tel quel, il montre une société qui, par un âge tardif au mariage, a limité sa descendance, avec les contraintes que l'on devine. La méthode utilisée qui repose sur la reconstitution des familles laisse de côté l'illégitimité ou la pratique du concubinage et n'a pas permis d'apprécier le taux de célibat ou celui des départs : il montre en tout cas qu'Ustaritz plus bourg que petite ville a recruté plus de la moitié de ses époux et donc de sa main d'œuvre en dehors des limites de la commune, même si beaucoup de ses hommes n'avaient pas fait plus de quinze kilomètres pour s'y rendre. 